\title{
Multidisciplinary Approach to Chronic Pelvic Pain
}

\author{
Erica C. Dun ${ }^{1}$, Vadim Morozov ${ }^{2}$ and Nisha A. Lakhi ${ }^{3}$ \\ ${ }^{1}$ Department of Obstetrics, Gynecology, \& Reproductive Sciences, Yale School of Medicine, New Haven, CT, USA \\ ${ }^{2}$ Department of Obstetrics, Gynecology, and Reproductive Sciences, University of Maryland School of Medicine, Baltimore, MD, USA \\ ${ }^{3}$ Department of Obstetrics E Gynecology, Richmond University Medical Center, Staten Island, NY, USA
}

Correspondence to:

Erica C. Dun, MD, MPH

Department of Obstetrics, Gynecology \&

Reproductive Sciences

Yale School of Medicine

New Haven, CT, USA

Tel: +1-203-737-4660

E-mail: erica.dun@yale.edu

Received: June 21, 2015

Accepted: July 15, 2015

Published: July 17, 2015

Citation: Dun EC, Morozov V, Lakhi NA. 2015. Multidisciplinary Approach to Chronic Pelvic Pain. JNeurol Exp Neurosci 1(1): 1-5.

Copyright: (C) 2015 Dun et al. This is an Open Access article distributed under the terms of the Creative Commons Attribution 4.0 International License (CC-BY) (http://creativecommons. org/licenses/by/4.0/) which permits commercial use, including reproduction, adaptation, and distribution of the article provided the original author and source are credited.

Published by United Scientific Group

\begin{abstract}
Chronic pelvic pain is a common medical condition affecting up to 16 percent of reproductive-aged women. There are many possible etiologies of chronic pelvic pain ranging from gynecologic to non-gynecologic. Treatment is based on the theorized cause but often refractory to traditional medical and surgical intervention. In over one third of the cases, the cause of chronic pelvic pain is unknown, even after an extensive workup including invasive surgical procedures. Women with chronic pelvic pain often have overlapping psychiatric diagnoses and history of physical or sexual abuse. Therefore, a multidisciplinary approach is recommended in order to address the patients' medical, psychological, and social needs.
\end{abstract}

\section{Keywords}

Chronic pelvic pain, Endometriosis, Interstitial cystitis, Irritable bowel syndrome, Multidisciplinary pain team

\section{Introduction}

Chronic pelvic pain (CPP) affects 4 to 16 percent of women [1,2]. The American College of Obstetricians and Gynecologists define it as "noncyclical pain of at least 6 months duration that appears in locations such as the pelvis, anterior abdominal wall, lower back, or buttocks, and that is serious enough to cause disability or lead to medical care" [3]. This common condition has significant social and economic impacts and substantial effects on health care utilization by women affected by this disorder. Outpatient costs of visits to physicians and mental health providers are estimated at $\$ 881.5$ million per year. Total indirect costs of chronic pelvic pain due to time lost from work are estimated at $\$ 555.3$ million per year [1].

There are many challenges to the diagnosis and treatment of chronic pelvic pain. The neurobiology of the pelvis is complex and our understanding of its pathophysiology is incomplete. The pelvic viscera are innervated by both the sympathetic and parasympathetic divisions of the autonomic nervous system, as well as by the somatic and sensory nervous systems [4]. The relationship between pain and tissue pathology is poorly understood. In fact, among women with chronic pelvic pain who undergo laparoscopy, approximately one-third have no obvious pelvic pathology [5]. Further, without a clear etiology, implementing a course of treatment is challenging. Even when an etiology is discovered, chronic pelvic pain is often refractory to traditional medical and surgical therapies.

In this review, we will review the most common causes of pelvic pain and 
discuss why chronic pelvic pain is best served through a multidisciplinary approach.

\section{Current known Etiologies of Chronic Pelvic Pain}

\section{Gynecologic causes}

Endometriosis is the most common diagnosis made at the time of laparoscopic evaluation for pelvic pain. It is found in approximately one third of the cases [6]. In fact, laparoscopy is the preferred method for diagnosis and treatment of even advanced endometriosis since the early 1990s [7]. Endometriosis is the implantation of uterine endometrium on ectopic sites such as the pelvic or abdominal peritoneum and viscera. These lesions can cause a chronic inflammatory disorder with increased number of macrophages and proinflammatory cytokines in the peritoneal fluid that lead to pain and infertility. However, neither the location nor amount of endometriosis correlates to the level of patients' pain $[8,9]$.

Up to 30 percent of women with pelvic inflammatory disease (PID) eventually develop CPP [10]. Though the underlying reason that PID leads to CPP is not clearly established, the predominant theory is the development of adhesive disease after acute infection and also tubal damage leading to hydrosalpinx.

Ovarian remnant also called residual ovary syndrome may occur in patients who have undergone bilateral oophorectomy. These patients subsequently present with symptoms due to ovulatory function from ovarian tissue inadvertently left behind. Typically patients with ovarian remnant syndrome have cyclic pelvic pain and a pelvic mass. Treatment involves resection of the ovarian remnant or hormonal suppression [11].

Adenomyosisis traditionally described as benign invasion of uterine endometrium into the myometrium, producing a diffusely enlarged uterus. Microscopically, it resembles ectopic non-neoplastic, endometrial glands and stroma surrounded by the hypertrophic and hyperplastic myometrium [12]. This condition causes abnormal uterine bleeding and dysmenorrhea. The pain is hypothesized to be due to the swelling and bleeding of the endometrial glands that grow into the uterine myometrium.

Fibroids, benign smooth muscle tumors of the uterus, can cause pressure symptoms and heavy bleeding depending on their size and location. Acute pain can occur if fibroids degenerate, twist, or expulse [13].

Pelvic congestion syndrome is a condition where pelvic varicosities or dilated uterine and ovarian veins are noted either radiologically or laparoscopically. One theory is that the damaged valves in the pelvic veins result in valvular incompetence leading to reflux and chronic dilation. Pelvic congestion is characterized by shifting location of pain, deep dyspareunia, post-coital pain, and exacerbation of pain after prolonged standing. However, dilated pelvic veins are a common finding in asymptomatic women as well [14].

\section{Genitourinary causes}

Interstitial cystitis (IC) or painful bladder syndrome (PBS) is an unpleasant sensation (pain, pressure, and discomfort) perceived to be related to the urinary bladder. It is associated with lower urinary tract symptoms of more than six weeks duration, in the absence of infection or other identifiable causes [15]. It is a chronic inflammatory condition that causes pelvic pain and irritable bladder dysfunction with exaggerated urinary frequency.

Other causes of chronic suprapubic pain are recurrent urinary tract infections for which a urethral diverticulum or source of vesicoureteral reflux should be investigated. Bladder neoplasia may also cause irritable bladder symptoms and should be suspected in women with hematuria, who smoke, and are over 60 years old.

\section{Gastrointestinal causes}

Irritable bowel syndrome (IBS) also referred to as visceral hyperalgesia is a pain syndrome characterized by chronic or intermittent abdominal pain associated with bowel function, in the absence of a physical cause [16]. Most patients have bowel dysfunction manifesting as diarrhea or constipation or both. Women are more than twice as likely to be diagnosed with IBS as men [17]. IBS is the most common diagnosis among women with chronic pelvic pain, occurring in $35 \%$ of this population [16].

Crohn's disease and ulcerative colitis are inflammatory bowel diseases (IBD) characterized by fatigue, diarrhea with abdominal pain, unanticipated weight loss, with or without rectal bleeding. Inflammatory processes cause bowel structures that lead to small and large bowel obstruction. Diverticulitis is another common cause of chronic abdominal or pelvic pain in older patients. Diverticular disease is most commonly located in the sigmoid colon, and the etiology is multifactorial with a combination of mucosal prolapse, fecal stasis, or localized ischemia leading to inflammation [18].

Chronic constipation and fecal impaction can lead to pelvic/abdominal pain. Causes may be congenital such as Hirschsprung's disease or environmental due to diet, chronic opioid use, and lifestyle choices. Celiac disease also called gluten intolerance, is an immune reaction to ingesting glutencontaining foods. Impaired absorption and digestion of nutrients by the small intestine results in weight loss, chronic diarrhea, and pain [19].

\section{Musculoskeletal system causes}

Multiple musculoskeletal conditions in the pelvis and abdomen can be grouped in to the category of chronic pelvic pain due to the location. Among the most common rheumatologic disorders that present with pelvic pain is fibromyalgia. Fibromyalgia is a poorly understood condition that is characterized by widespread musculoskeletal pain, accompanied by fatigue, cognitive disturbances, psychiatric syndromes, and somatic symptoms. On physical examinations, tenderness is usually present in soft-tissue anatomic locations. Three criteria are required for the diagnosis of fibromyalgia: 1) Elevated scores on the Widespread Pain Index and Symptom Severity, 2) Symptoms present for at least 3 months, 3) No 
other disorder which would explain the pain [20].

Myofascial pain syndrome (MFPS) describes a disorder in which pain is attributed to tender pelvic floor muscles that are exacerbated by hypersensitive trigger points in the skeletal muscle [21]. Symptoms include a sense of aching, heaviness, or burning in the pelvis, vagina, vulva, rectum, or bladder, and referred pain to the thighs, buttocks, or lower abdomen. The etiology of MFPS is unclear, as pelvic organ pathology, neuromuscular disorders, and psychologic conditions can all contribute.

Chronic abdominal wall pain is a chronic pain emanating from the abdominal wall and it is frequently confused with visceral pain. The characteristic feature of chronic abdominal wall pain is localization of pain to a highly discrete region of the abdomen. The cause of the pain is cutaneous nerve entrapment from the neurovascular bundles of T7 to T12 caused by intra- or extra-abdominal pressure or localized scarring [22]. Cyclic abdominal wall pain in reproductive-aged women with prior abdominal surgery may suggest abdominal wall endometriosis. Endometriosis in the scar tissue of abdominal incisions, including laparoscopic port sites, hernia repair, and laparotomy incisions and have been estimated to range from $0.04 \%$ to $12 \%$ in small cohorts of patients surgically treated for endometriosis [23].

Osteitis pubis is an idiopathic inflammatory disease of the pubic symphysis and its surrounding attachments including cartilage, ligaments, muscles, and the pubic rami. People who are most predisposed to this condition are athletes who play soccer, football, ice hockey, and rugby [24, 25]. The mechanism is thought to be due to repetitive movements in conjunction with opposing shearing forces across the pubic symphysis. Non-sport conditions such as rheumatologic diseases, pregnancy, pelvic trauma, and pelvic surgery are also associated with osteitis pubis [26].

\section{Mental health causes}

Various mental health issues such as somatization, drug dependency, physical and sexual abuse, anxiety and depression are associated with chronic pelvic pain in women. Somatization is a syndrome of physical symptoms that involve more than one part of the body, but no physical cause can be found after the patient has been worked up. Patients with chronic pain are more likely to manifest a dependency to narcotic pain medication. In addition, they have a decreased response to opioids and require higher than average doses for adequate analgesia [27]. Women with pelvic pain have a higher incidence of prior physical or sexual abuse. Up to $60 \%$ of women who have been physically and sexually abused reported new or worsening pain symptoms [28]. Pain severity has been linked to psychological symptoms, specifically anxiety and depression [29]. Gomide et al. noted a positive association between pain severity in CPP patients and psychological distress [30]. These conditions occur more frequently in patients with pelvic pain; however it is unclear if they are causally related.

In over one-third of patients, the etiology of the chronic pelvic pain is unknown even though an exhaustive workup including laparoscopy has been performed [5].

\section{Multidisciplinary Care}

Over 40 years ago, Fordyce advocated for a multidisciplinary treatment program for patients with chronic non-malignant pain [31]. He identified that patients with chronic pain exhibited more maladaptive behaviors such as excessive reliance on medication, bed rest, and a restricted life-style, while not engaging in physical activity, socializing, or fulfilling home and work responsibilities. He promoted an operant conditioning model to change behavior and reduce the maladaptive behaviors. His work paved the way for the current cognitive-behavioral paradigms with the goals of modifying the patients' thoughts and beliefs about pain and working towards realistically managing pain. The growing acceptance of the cognitive-behavioral model for the treatment of chronic pelvic pain naturally lends to collaborating with health care professionals from different disciplines to treat patients with chronic pelvic pain.

Chronic pain is a difficult condition to manage, and often resistant to traditional medical and surgical management. The multidisciplinary model for pelvic pain is adapted based on interdisciplinary programs for other types of chronic pain. Kames et al. reported that women with chronic pelvic pain who completed a 6-8 week multidisciplinary program that included somatic and behavioral therapies showed significant decreased in reported levels of pain, anxiety, and depression compared to controls who had standard medical and surgical treatments. In addition, psychosocial functioning improved including return to work, increased social activities, and improved sexual activity in the women treated with an interdisciplinary approach [32]. Flor et al. conducted a meta-analysis of 65 studies (3089 patients) on the efficacy of multidisciplinary treatments for chronic back pain. He showed that multidisciplinary treatment was superior to unimodal treatment and no treatment, with beneficial effects maintained over a period of time [33].

\section{Elements of a Multidisciplinary Team}

The multidisciplinary team was created on the premise that chronic pain is a complex psycho physiological condition requiring both somatic and psychological treatment. The benefit of the multidisciplinary approach is that it emphasizes to the patient the importance of all aspects of the pain experience and the contributions of all members of the pain team. The aims of the multidisciplinary clinic should be established by the members, and one common goal may be to relieve pain if possible, but also reduce physical and psychological dysfunction in order to improve quality of life.

The primary team typically consists of a gynecologist, psychologist, and anesthesiologist with expertise in pain management, and physiotherapist. The gynecologist's role is to explore the nature of the symptoms in greater detail in order to determine if the pain is due to an organic gynecological pathology. They have a duty to explain the nature of the diagnosis and treatment that can be understood by the patients. After thoughtful workup, diagnostic laparoscopy can be undertaken to attempt to discover the etiology of the pain; however repeated surgical interventions are discouraged 
because chronic pain often follows a cycle of exacerbation and remission. Thus, if surgery takes places during an acute phase and the pain resolves, then the intervention will be regarded to be beneficial and successful by the patient. This then leads to the persistence of the patient in seeking further surgical procedures.

An important member of the primary team who should be at the patient' first assessment is the psychologist. Chronic pain patients are often reluctant to see a psychologist for a problem they feel is physical. Thus, the psychologist can be introduced as a specialist who understands how the problem of chronic pain can affect people's lives and help them cope with pain. A thorough psychosocial and behavioral assessment is an essential part of the evaluation of chronic pelvic pain. The focus should be on identifying factors other than physical factors that cause the pain symptoms and determining the impact the pain has on the women's emotional wellbeing, relationships, and daily functioning.

The anesthesiologist with a focus in pain management is a critical member of the team because of their knowledge of acute and chronic somatic, visceral and neuropathic pain. They are well versed in the treatment of opioid and non-opioid analgesic drugs and are able to perform diagnostic, prognostic, and therapeutic nerve blockades.

Musculoskeletal dysfunction is major causative factor in chronic pelvic pain due to repetitive stress and strain associated with faulty posture, poor body mechanics and physical fitness. Thus, the physiotherapist's role is to assess and manage musculoskeletal dysfunction through education, therapeutic exercise, stretching, manual myofascial release, muscle retraining, and mobilization.

Finally the nurse-coordinator has an essential role as a point of contact for the patient and members of the multidisciplinary team. Additionally, they are a vital educator and can address some questions that patients may not want to discuss with their doctors. A nurse specialist can often explain and discuss issues with the patient in a more relaxed and comfortable setting.

On occasion, it may be necessary to refer patients for further specialist opinion after initial evaluation. Therefore, attaining collaborations with a clinical nurse specialist, social worker, gastroenterologist, urologist, orthopedic surgeon, and psychiatrist with an interest in pain management are important in order to provide comprehensive care for patients with chronic pelvic pain.

\section{Outcome Measures}

Important technical aspects of the clinic that should be discussed ahead of time are whether the clinic will be a primary or tertiary referral center and whether all the healthcare professionals will see the patient at the same time or individually. Creating an information booklet on causes and treatment for pelvic pain is a helpful educational tool for patients. Questionnaires can assist with gathering health histories and assessing treatment success. Developing an efficient workflow is important for the patient to remain engaged and prevent fatigue and frustration. A 45 to 60 minute appointment is usually sufficient for evaluation and initiation of treatment. Treatment decisions should be discussed by the team and a cohesive plan made and discussed with the patient.

Along with establishing the aims of the multidisciplinary clinic, outcome measures need to be complete in order to monitor the patient's progress and the program's success. Pain intensity can be measured with visual analog scales. Depression and anxiety scales can assist with assessing the psychosocial aspects of pain management. Other objective outcome measures are the drug usage, health care utilization, return to work, return to activities of daily living, and quality of life assessments.

\section{Conclusion}

Chronic Pelvic Pain is a multifaceted disorder. It is a challenge to health care providers because of its unclear etiology and complex natural history. The multifactorial nature of chronic pelvic pain should be discussed and explored with patients at their initial visit, as a variety of gynecologic, gastrointestinal, urologic, musculoskeletal and psychiatric disorders can be attributed to chronic pelvic pain syndromes. The initial history should include questions about the pattern of the pain, and its association with other problems, such as psychological factors, bladder and bowel symptoms, the effect of movement and posture on the pain, or other gynecological manifestations. Patients should be managed by a multidisciplinary team that incorporates the skills of a gynecologist, psychologist, anesthesiologist, urologists, gastroenterologist, physiotherapist and nurse-coordinator. In this manner, both organic and psychological aspects of the patients' pain can be addressed. Treatment plans should be patient centered and incorporate views from all specialists.

\section{References}

1. Mathias SD, Kuppermann M, Liberman RF, Lipschutz RC, Steege JF. 1996. Chronic pelvic pain: prevalence, health-related quality of life, and economic correlates. Obstet Gynecol 87(3): 321-327. doi: 10.1016/00297844(95)00458-0

2. Zondervan KT, Yudkin PL, Vessey MP, Dawes MG, Barlow DH, et al. 1999. Prevalence and incidence of chronic pelvic pain in primary care: evidence from a national general practice database. BrJ Obstet Gynaecol 106(11): 1149-1155. doi: 10.1111/j.1471-0528.1999.tb08140.x

3. Yunker A, Sathe NA, Reynolds WS, Likis FE, Andrews J, et al. 2012. Systematic review of therapies for noncyclic chronic pelvic pain in women. Obstet Gynecol Surv 67(7): 417-425. doi: 10.1097/ OGX.0b013e31825cecb3

4. Lamvu G, Steege JF. 2006. The anatomy and neurophysiology of pelvic pain. J Minim Invasive Gynecol 13(6): 516-522. doi: 10.1016/j. jmig.2006.06.021

5. Howard FM. 1993. The role of laparoscopy in chronic pelvic pain: promise and pitfalls. Obstet Gynecol Surv 48(6): 357-387.

6. Howard FM. 1996. The role of laparoscopy in the evaluation of chronic pelvic pain: pitfalls with a negative laparoscopy. $J$ Am Assoc Gynecol Laparosc 14(1): 85-94. doi: 10.1016/S1074-3804(96)80116-2

7. Nezhat C, Nezhat F, Nezhat CH, Seidman DS. 1995. Severe endometriosis and operative laparoscopy. Curr Opin Obstet Gynecol 7(4): 299-306.

8. Hsu AL, Sinaii N, Segars J, Nieman LK, Stratton P. 2011. Relating pelvic pain location to surgical findings of endometriosis. Obstet Gynecol 


\section{8(2 Pt 1): 223-230. doi: 10.1097/AOG.0b013e318223fed0}

9. Fauconnier A, Chapron C, Dubuisson JB, Vieira M, Dousset B, et al. 2002. Relation between pain symptoms and the anatomic location of deep infiltrating endometriosis. Fertil Steril 78(4): 719-726. doi: 10.1016/S0015-0282(02)03331-9

10. Ness RB, Soper DE, Holley RL, Peipert J, Randall H, et al. 2002. Effectiveness of inpatient and outpatient treatment strategies for women with pelvic inflammatory disease: results from the Pelvic Inflammatory Disease Evaluation and Clinical Health (PEACH) Randomized Trial. Am J Obstet Gynecol 186(5): 929-937. doi: 10.1067/mob.2002.121625

11. Nezhat CH, Seidman DS, Nezhat FR, Mirmalek SA, Nezhat CR. 2000. Ovarian remnant syndrome after laparoscopic oophorectomy. Fertil Steril 74(5): 1024-1028. doi: 10.1016/S0015-0282(00)01604-6

12. Benagiano G, Habiba M, Brosens I. 2012. The pathophysiology of uterine adenomyosis: an update. Fertil Steril 98(3): 572-579. doi: 10.1016/j.fertnstert.2012.06.044

13. Stewart EA. 2015. Clinical practice. Uterine fibroids. $N$ Engl J Med 372(17): 1646-1655. doi: 10.1056/NEJMcp1411029

14. Rozenblit AM, Ricci ZJ, Tuvia J, Amis ES Jr. 2001. Incompetent and dilated ovarian veins: a common CT finding in asymptomatic parous women. AJR Am J Roentgenol 176(1): 119-122.

15. Hanno PM, Erickson D, Moldwin R, Faraday MM. 2015. Diagnosis and Treatment of Interstitial Cystitis/Bladder Pain Syndrome: AUA Guideline Amendment. J Urol 193(5): 1545-1553. doi: 10.1016/j. juro.2015.01.086

16. Williams RE, Hartmann KE, Sandler RS, Miller WC, Savitz LA, et al. 2005. Recognition and treatment of irritable bowel syndrome among women with chronic pelvic pain. Am J Obstet Gynecol 192(3): 761-767. doi: 1016/j.ajog.2004.10.634

17. Wilson S, Roberts L, Roalfe A, Bridge P, Singh S. 2004. Prevalence of irritable bowel syndrome: a community survey. Br J Gen Pract 54(504): 495-502.

18. Abraham C, Cho JH. 2009. Inflammatory bowel disease. N Engl J Med 361(21): 2066-2078. doi: 10.1056/NEJMra0804647

19. Porpora MG, Picarelli A, Prosperi Porta R, Di Tola M, D'Elia C, et al. 2002. Celiac disease as a cause of chronic pelvic pain, dysmenorrhea, and deep dyspareunia. Obstet Gynecol 99(5 Pt 2): 937-939. doi: 10.1016/ S0029-7844(01)01711-2

20. Jones GT, Atzeni F, Beasley M, Fluss E, Sarzi-Puttini P, et al. 2015. The prevalence of fibromyalgia in the general population: a comparison of the American College of Rheumatology 1990,2010, and modified 2010 classification criteria. Arthritis Rheumatol 67(2): 568-575. doi: 10.1002/ art.38905
21. Srinivasan AK, Kaye JD, Moldwin R. 2007. Myofascial dysfunction associated with chronic pelvic floor pain: management strategies. Curr Pain Headache Rep 11(5): 359-364. doi: 10.1007/s11916-007-0218-0

22. Srinivasan R, Greenbaum DS. 2002. Chronic abdominal wall pain: a frequently overlooked problem. Practical approach to diagnosis and management. Am J Gastroenterol 97(4): 824-830. doi:10.1111/j.15720241.2002.05662.x

23. Ecker AM, Donnellan NM, Shepherd JP, Lee TT. 2014. Abdominal wall endometriosis: 12 years of experience at a large academic institution. Am J Obstet Gynecol 211(4): 363. doi: 10.1016/j.ajog.2014.04.011

24. Holt MA, Keene JS, Graf BK, Helwig DC. 1995. Treatment of osteitis pubis in athletes. Results of corticosteroid injections. Am J Sports Med 23(5): 601-606. doi: 10.1177/036354659502300515

25. Karpos PA, Spindler KP, Pierce MA, Shull HJ Jr. 1995. Osteomyelitis of the pubic symphysis in athletes: a case report and literature review. Med Sci Sports Exerc 27(4): 473-479.

26. Vincent C. 1993. Osteitis pubis. J Am Board Fam Pract 6(5): 492-496. doi: $10.3122 /$ jabfm.6.5.492

27. Steele A. 2014. Opioid use and depression in chronic pelvic pain. Obstet Gynecol Clin North Am 41(3): 491-501. doi: 10.1016/j.ogc.2014.04.005.

28. Ulirsch JC, Ballina LE, Soward AC, Rossi C, Hauda W, et al. 2014. Pain and somatic symptoms are sequelae of sexual assault: results of a prospective longitudinal study. Eur J Pain 18(4): 559-566. doi: 10.1002/j.1532-2149.2013.00395.x

29. Miller LR, Cano A, Wurm LH. 2013. A motivational therapeutic assessment improves pain, mood, and relationship satisfaction in couples with chronic pain. J Pain 14(5): 525-537. doi: 10.1016/j. jpain.2013.01.006

30. Gomide L, de Souza Montenegro M, Nogueira A, poli O, Silva J, et al. 2012. Lower pain thresholds in women with chronic pelvic pain: recognizing the role of anxiety and depression as part of personcentered approaches to treatment. Int J Person Cent Med 2(2): 271-278. doi: $10.5750 /$ ijpcm.v2i2.225

31. Fordyce WE, Fowler RS Jr, Lehmann JF, Delateur BJ, Sand PL, et al. 1973. Operant conditioning in the treatment of chronic pain. Arch Phys Med Rehabil 54(9): 399-408.

32. Kames LD, Rapkin AJ, Naliboff BD, Afifi S, Ferrer-Brechner T. 1990. Effectiveness of an interdisciplinary pain management program for the treatment of chronic pelvic pain. Pain. 41(1): 41-46. doi: 10.1016/03043959(90)91107-T

33. Flor H, Fydrich T, Turk DC. 1992. Efficacy of multidisciplinary pain treatment centers: a meta-analytic review. Pain 49(2): 221-230. doi: 10.1016/0304-3959(92)90145-2 